\title{
HIFU is effective for unresectable HCC
}

Patients with hepatocellular carcinoma (HCC) have high morbidity and mortality. High-intensity focused ultrasound (HIFU) is a local ablation therapy that, unlike other standard ablation methods, does not puncture the tumor; therefore, it is not associated with the risk of bleeding and cancer cell dissemination. Although ablation rates as high as $68 \%$ have been reported with this relatively new technique, it is not clear what risk factors affect the technique or how they affect survival outcomes.

In a single-center study, Sheung Tat Fan and colleagues report on the risk factors and outcomes from 49 patients with unresectable HCC. "The advantage of HIFU is that it can be applied to tumors associated with poor liver function and ascites. It may be a good method to treat HCC patients while they are waiting for liver grafts," explains Fan.

Tumor size was the only risk factor to significantly affect the primary technique; patients with the best outcomes had smaller tumors than those with residual tumors after HIFU treatment, with $3 \mathrm{~cm}$ as the cut-off value for increased risk. The overall recurrence rate was $61.9 \%$, which compares favorably to other studies. The primary effectiveness rate for patients treated with HIFU alone was $89.2 \%$ compared with $66.6 \%$ in patients who received pretreatment lipiodol deposition, which is comparable to radiofrequency ablation. The 1-year and 3 -year overall survival rates were $87.7 \%$ and $62.4 \%$. Child-Pugh grade was the only significant prognostic factor that affected overall survival. Fan concludes: "HIFU treatment can be considered as one of the effective treatment options in the setting of current tumor ablation technology. This technique is being studied as an adjunct in patients requiring liver transplantation".

\section{Lisa Hutchinson}

Original article Ng, K. et al. High-intensity focused ultrasound for hepatocellular carcioma: a single-center experience. Ann. Surg. 253, 981-987 (2011). 